\title{
ASYMPTOTIC DESCRIPTION AND THE DIAGNOSTIC PROPERTIES OF LOW-DEGREE STELLAR P-MODES
}

\author{
I.W. ROXBURGH ${ }^{1}$ AND S.V. VORONTSOV ${ }^{1,2}$ \\ ${ }^{1}$ Astronomy Unit, Queen Mary and Westfield College \\ Mile End Road, London E1 4NS, UK; \\ ${ }^{2}$ Institute of Physics of the Earth \\ B.Gruzinskaya 10, Moscow 123810, Russia
}

\begin{abstract}
Standard asymptotic descriptions of stellar p-modes use $1 / \omega$ as a small parameter; either the degree $\ell$ is kept constant in the asymptotic expansions (e.g. Tassoul 1980), or the parameter $\tilde{w}=(\ell+1 / 2) / \omega$, which specifies the position of the inner turning point (e.g. Vorontsov 1991). At low degree, due to the strong effects of gravity perturbations, these expansions are known to produce rather poor results, even when developed to higher order (Roxburgh and Vorontsov 1994). Here, we employ an alternative asymptotic expansion, capable of providing much better accuracy at low degree-with $\tilde{w}$ as a small parameter instead of $1 / \omega$, and test its diagnostic capabilities.
\end{abstract}

The asymptotic eigenfrequency equation, which includes terms of order $\tilde{w}^{2}$, is

$$
F_{0}(\omega)+\tilde{w}^{2} F_{2}(\omega) \simeq \frac{\pi}{\omega}[n+\ell / 2+1 / 4+\alpha(\omega)],
$$

where $n$ is radial order and $\alpha(\omega)$ is the surface phase shift. When terms of order $\tilde{w}^{2}$ are neglected, the frequencies degenerate with respect to $n+\ell / 2$; the function $F_{0}(\omega)$ is an effective acoustic radius. Small frequency separations are described by $F_{2}(\omega)$ :

$$
F_{2}\left(\omega_{\ell, n}\right) \simeq \frac{\pi}{2}\left(n+\frac{\ell}{2}+\frac{1}{4}\right) \frac{\omega_{\ell, n}-\omega_{\ell+2, n-1}}{2 \ell+3} .
$$

After $F_{2}(\omega)$ has been measured and approximated by linear regression $F_{2}(\omega) \simeq A+$ $B \omega$, the surface term $\alpha(\omega)$ in its combination with $F_{0}(\omega)$ can be inferred as

$\beta^{*}(\omega)=\omega^{2} \frac{d}{d \omega}\left[\frac{F_{0}(\omega)}{\pi}-\frac{\alpha(\omega)}{\omega}\right] \simeq \frac{\bar{\omega}}{\omega_{\ell, n+1}-\omega_{\ell, n}}-n-\frac{\ell}{2}-\frac{3}{4}+\frac{\left(\ell+\frac{1}{2}\right)^{2}}{\pi \bar{\omega}}(2 A+B \bar{\omega})$,

at frequency $\bar{\omega}=\left(\omega_{\ell, n+1}+\omega_{\ell, n}\right) / 2$. Numerical results are illustrated by Figs 1 and 2 . 


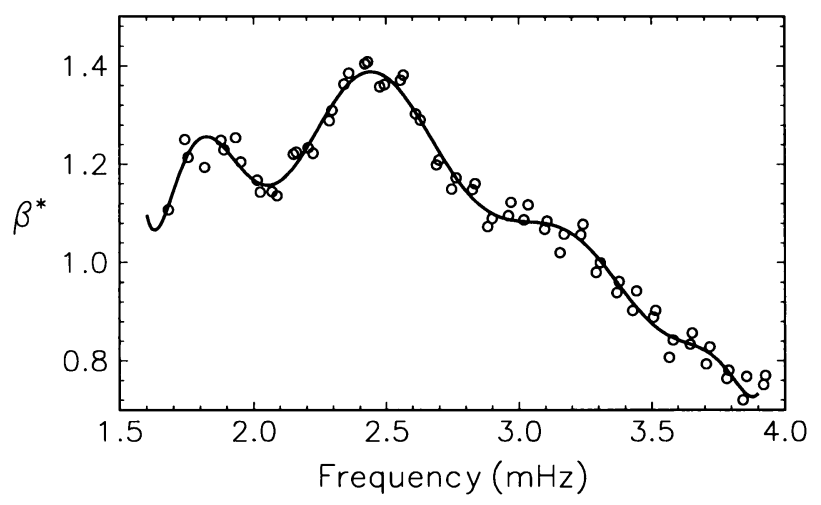

Figure 1. Function $\beta^{*}(\omega)$ inferred from the solar eigenfrequencies of degree $\ell$ from 0 to 3 . The solid line shows a best-fit polynomial of degree 10 . The prominent quasi-periodic variation with frequency is produced by the HeII ionization region

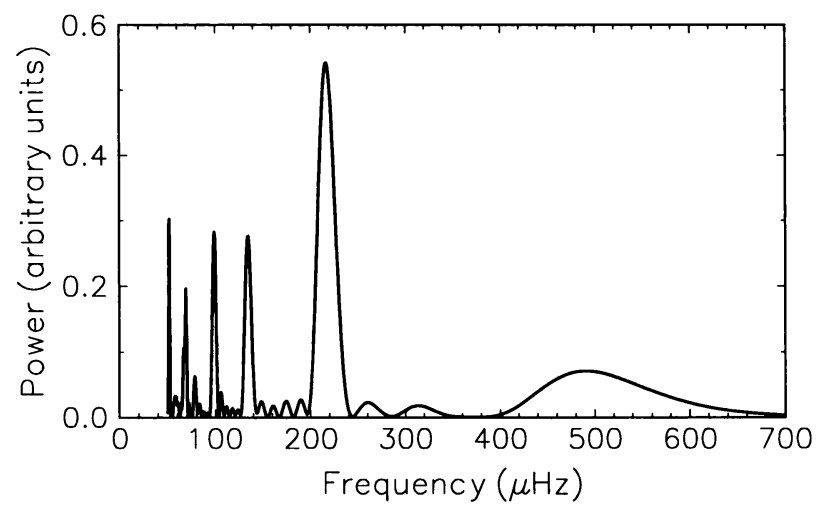

Figure 2. Fourier transform of the approximation residuals seen in Fig.1. The prominent peak at about $220 \mu \mathrm{Hz}$ is produced by the rapid variation of the sound-speed gradient at the base of the solar convection zone

\section{References}

Tassoul, M. (1980), ApJS 43, p.469

Roxburgh, I. W. and Vorontsov, S. V. (1994), Mon. Not. R. astr. Soc. 268, p.143

Vorontsov, S. V. (1991), SvA 35, p.400 